\title{
El contexto cultural en la traducción de la LIJ: la adaptación Cuentos para contar de Naguib Mahfuz
}

\author{
(The cultural context in the translation of young people's \\ literature: the adaptation of Naguib Mahfuz's Tales for telling)
}

\author{
SheRINE SAMY GAMAL ELDIN \\ rouna81@hotmail.com \\ Universidad de Granada
}

Fecha de recepción: 28 de mayo de 2012

Fecha de aceptación: 1 de junio de 2012

Resumen: En este artículo, se defiende la importancia que tiene el contexto cultural en la traducción de la literatura infantil y juvenil (LIJ). El estudio analiza, desde un punto de vista comparativo-contrastivo, la adaptación infantojuvenil Cuentos para contar, realizada en español a partir del original árabe para adultos حكايات حارتنا (Hikayat haratina), del Nobel egipcio Naguib Mahfuz, pero con la mediación de una traducción anterior al español igualmente para adultos: Historias de nuestro barrio. Para la clasificación y análisis de los marcadores culturales, seguimos el método de Gisela Marcelo Wirnitzer (2007), aunque adaptándolo a las características de la obra analizada. Se hace especial hincapié en el contexto cultural del árabe y en cómo se ha trasladado a la adaptación española. El objetivo último de este trabajo es mostrar la ambigüedad que se ha producido a veces en la adaptación, destinada a lectores adolescentes españoles, por un tratamiento poco adecuado de los marcadores culturales.

Palabras clave: Marcadores culturales, Literatura/Adaptación infantil y juvenil, Naguib Mahfuz, Cuentos para contar.

Abstract: In this paper, I uphold the importance of the cultural context in the translation of Children's Literature. From a comparative-contrastive point of view, I study the children's adaptation -Cuentos para contar- translated to Spanish from the Arabic adult's original work حكايات حارتنا (Hikayat haratina), written by the Egyptian Nobel Prize winner Naguib Mahfouz, but carried out through the mediation of a previous translation to Spanish also carried out for adults - Historias de nuestro barrio-. For the classification and analysis of cultural markers, I chose to follow Gisela Marcelo Wirnitzer's Methodology (2007), though adapting it according to the analyzed work's characteristics. This study puts special emphasis on the cultural context of the Arabic original work and how it was transferred to the Spanish adaptation. My ultimate aim here is to highlight the ambiguity which has occurred sometimes in the adapted work, bound for Spanish young-adult readers, through a not-so-adequate treatment of the cultural markers.

Key words: Cultural markers, Children's literature/adaptation, Naguib Mahfuz, Cuentos para contar. 


\section{INTRODUCCIÓN}

En el presente trabajo, nos centramos en los marcadores culturales, un aspecto de gran importancia en el campo de la traducción literaria y, muy especialmente, en el de la traducción de la LIJ. La novedad de este estudio no reside tanto en el tema como en la especificidad de la obra seleccionada: se trata de la adaptación infantojuvenil española Cuentos para contar, realizada a partir de un original árabe para adultos حكابات حارتنا (Hikayat haratina), pero con la mediación de otra traducción, anterior a la adaptación y obra de la misma traductora, Historias de nuestro barrio, que iba dirigida, como el original, al público adulto.

La elección de este tema se ha inspirado en el artículo "Mercado y traducción: Naguib Mahfuz para niños" de Juan Pablo Arias Torres, que fue publicado en la colección Traducción y literatura infantil en 2003 y en el cual se estudia esta misma adaptación infantojuvenil, Cuentos para contar. En ese artículo, Arias Torres comenta el éxito que alcanzó Mahfuz tras haber sido galardonado con el Premio Nobel de Literatura, hasta el punto de llevar a la Editorial Litertarias/Prodhufi a tomar la iniciativa de presentarlo a los lectores adolescentes españoles. Para apoyar su argumento, el investigador español cuestiona los aspectos paratextuales de la adaptación (su publicación, los criterios de selección de los cuentos incluidos, las notas a pie de página, los títulos añadidos, el cambio de título de la adaptación respecto de la primera traducción para adultos, etc.), pero en ningún momento trata los aspectos textuales o lingüísticos, por lo que hemos decidido desarrollarlos en este trabajo.

En primer lugar, y antes de comenzar nuestro estudio traductológico, ofrecemos una breve información sobre el autor y su obra. En 1975, vio la luz حكايات حارتنا (Hikayat haratina), una colección de 78 cuentos cortos de Naguib Mahfuz, escritor egipcio que por aquel entonces era conocido prácticamente sólo en Egipto y en el mundo árabe. En octubre de 1988, Mahfuz obtuvo el Premio Nobel de Literatura, la primera y hasta ahora única ocasión en que un escritor árabe lo ha recibido. Por lo tanto, este año podría considerarse como el del comienzo del boom internacional de este escritor; de hecho, antes de que acabara ese año, podían encontrarse en las librerías españolas tres novelas traducidas de Mahfuz y en el transcurso del año siguiente aparecieron otras siete, entre ellas Historias de nuestro barrio, traducción de Hikayat haratina, publicada en mayo de 1989 en la Editorial Litertarias/Prodhufi y realizada por la traductora María Rosa de Madariaga. Unos meses más tarde, concretamente en noviembre de 1989, la misma editorial decide lanzar al mercado una adaptación infantojuvenil de esta obra árabe, en traducción también de María Rosa de Madariaga, que se publica con el título de Cuentos para contar, pero incluye solamente cinco cuentos de los 78 del original. 
Los criterios de selección de los cinco cuentos no se especifican. No parece que el principal criterio haya sido el temático, porque los cuentos elegidos no presentan un asunto diferente del resto. Tampoco la extensión, pues, salvo un cuento, los otros cuatro ocupan cuatro páginas, característica que comparten cinco cuentos más del original árabe. En una consulta (por comunicación personal) realizada por Juan Pablo Arias Torres para conocer el criterio de selección adoptado, un miembro del Departamento Literario de la Editorial Libertarias/Prodhufi le informó de que los relatos fueron "seleccionados por nuestro equipo editorial bajo el criterio de que eran los apropiados para jóvenes adolescentes destinatarios de la colección" (2003: 126).

Nos parece importante recordar un hecho que subraya Arias Torres en su artículo y es que en Cuentos para contar "no hay encargo de traducción" (2003: 125). Por nuestra parte, tras haber mantenido una conversación telefónica con un responsable de la Editorial Libertarias/Prodhufi, se nos informó de que le pareció una idea interesante introducir a Naguib Mahfuz en el mundo de los adolescentes españoles, lo que también nos confirmó la traductora en respuesta a una consulta que le hicimos por correo electrónico. En ella, nos decía que la idea había sido del editor: "me preguntó si estaba de acuerdo con que se hiciera una selección de algunas historias para incluirlas en un volumen destinado a los niños y la idea me gustó". Recordemos aquí que el original árabe, حكايات حارتنا (Hikayat haratina), fue traducido íntegramente por la misma traductora de la adaptación en una versión titulada Historias de nuestro barrio, que iba dirigida como el texto original al público adulto. En resumen, lo que queremos subrayar es que la adaptación infantojuvenil no es una nueva traducción. Al contrario, se llevó el texto español -y con 'texto' nos referimos exclusivamente al 'componente lingüístico'- desde la primera versión dirigida a lectores adultos y se trasladó directamente a una segunda versión para lectores adolescentes, difiriendo apenas la segunda versión de la primera en el tratamiento de los aspectos paratextuales (nuevo diseño, ilustraciones, tamaño y fuente de letra distintos y, cómo no, cambio radical del título); pero, repetimos, sin modificar nada el aspecto lingüístico.

Una vez aclaradas estas cuestiones, presentamos a continuación una explicación de lo que entendemos aquí por 'marcadores culturales' y el tipo de intervención que debería realizar el traductor.

\section{LOS MARCADORES CULTURALES Y EL INTERVENCIONISMO DEL TRADUCTOR PARA SU TRADUCCIÓN}

Una de las cuestiones que un traductor de LIJ debe tener muy en cuenta a la hora de iniciar su tarea son los 'marcadores culturales', puesto 
que estos fenómenos suelen manifestarse dentro de los textos literarios como interferencias, obstaculizando su comprensión.

Los estudiosos han denominado a estos elementos de varias formas: Nida (1964) los llama cultural features; Newmark (1982) usa la expresión cultural terms; tanto Bódeker y Fresse (1987) como Koller (1992) recurren al término realia; Nord (1994), por su parte, aplica la denominación culturalmarker; Franco (1996) utiliza elementos culturales específicos; Mayoral (1994) los nombra como referencias culturales; Mayoral y Muñoz (1997) los denominan segmentos textuales marcadamente culturales, etc. (cit. en Herrero 2000:311). En nuestro trabajo, usaremos indistintamente 'marcadores', 'referencias', 'elementos' y 'fenómenos culturales' para evitar repeticiones excesivas de alguno de estos términos, considerándolos, por consiguiente, todos iguales en su significado.

En cuanto al concepto de 'cultura', ha habido infinidad de definiciones, pero ninguna comúnmente aceptada ni que cubra todo el concepto. Por lo que se refiere a los objetivos de este trabajo, la definición de Gisela Marcelo Wirnitzer (2007: 49) nos parece una de las definiciones más completas:

la cultura es el conjunto heredado de conocimientos, creencias, costumbres, normas y reglas de comportamiento compartido y heredado por todos los miembros de una comunidad, y que les condiciona para poder actuar adecuadamente dentro de la sociedad de la que forman parte. La cultura les permite al mismo tiempo juzgar, ver y percibir la realidad, comportarse y saber qué se espera de ellos como miembros de esa comunidad. Incluye, además, aquellos objetos materiales producidos en ella, así como los medios necesarios para producir y manejar dichos objetos como productos de una evolución. A través de las culturas, se distinguen unas comunidades de otras y las diferencias entre ellos se manifiestan en sus productos, como por ejemplo, en su literatura.

A partir de estas consideraciones en torno al concepto de 'cultura', han surgido a su vez distintas definiciones que pretenden explicar lo que se entiende por 'marcador o referencia cultural': para Leticia Herrero (2000: 311) las referencias culturales son "elementos cuyo contenido muestra potencialmente cierta resistencia a la transferencia"; Marisa Presas (2000), por su parte, las define como "the verbal expression of a phenomenon that is specific to a given culture" (cit. en Marcelo Wirnitzer 2007: 74). No obstante, Roberto Mayoral (1994) presenta una definición muy adecuada para nuestro trabajo, relacionando las referencias culturales con la traducción: 
Por referencias culturales entendemos los elementos del discurso que por hacer referencia a particularidades de la cultura de origen no son entendidos en absoluto o son entendidos en forma parcial o son entendidos de forma diferente por los miembros de la cultura de término (Mayoral 1994: 76).

Podríamos decir, a modo de resumen, que los marcadores culturales son "aquellos elementos lingüísticos que reflejan una forma de pensar, de sentir, de actuar, de ver la realidad, condicionados por la cultura de la que forman parte y en la que han sido producidos así como hechos y productos materiales de dicha cultura" (Marcelo Wirnitzer 2007: 77-78). Constituyen referencias o marcadores culturales los nombres propios, los topónimos, los nombres institucionales, las unidades de peso y de medida, las monedas, el folclore, las referencias históricas, las comidas y los hábitos alimenticios, el humor, los tabúes, etc.

Un concepto que también nos interesa recordar es el de la 'intertextualidad'. Acuñado por Julia Kristeva ([1965] 1969), esta lingüista búlgara lo define como "todo texto que se construye como un mosaico de citas y es también la absorción y transformación de otro texto" (Kristeva 1997: 3). En otras palabras, la intertextualidad es la presencia, con mayor o menor grado de explicitación, de textos o contextos precedentes en un texto determinado. Belén González Cascallana (2006: 98) argumenta que, a través de la intertextualidad, un texto ya no es visto como un contenedor de significados, sino como un espacio intertextual en el que varios elementos se combinan, se absorben y se transforman. Octavio Paz (1971: 38), por su parte, sugiere que todos los textos son "translations of translations of translations", ya que todo texto remite a otros textos que lo preceden y lo rodean. De la misma forma, Theo Hermans (1993: 78) asimila la intertextualidad al "intercultural traffic", refiriéndose al contexto sociocultural en el que se hallan los textos y el cual los condiciona fuertemente (cit. en González Cascallana 2006: 99).

En nuestro trabajo, consideramos la 'intertextualidad' como parte integrante de los marcadores culturales. En ella incluimos las referencias que, para ser entendidas, remiten a otros textos o contextos anteriores establecidos en la cultura tradicional egipcia.

La importancia de la traducción de los marcadores culturales reside en el hecho de que lo que se busca es la aceptabilidad del texto traducido en la cultura meta (CM), por lo cual lo que ha de conseguir es la aceptación y entendimiento del libro por parte del lector infantil, evitando en lo posible que se aburra o que desista de su lectura. En otras palabras, el traductor de LIJ debe conocer las dos culturas, la original de la que parte y la meta a la que se dirige, además de ser consciente de las diferencias entre ambas para luego poder tomar las decisiones adecuadas sobre las estrategias 
traslativas que adoptará con el fin de que el texto consiga su objetivo: ser aceptado por sus futuros lectores.

Siguiendo con los marcadores culturales, nos parece relevante matizar dos conceptos muy relacionados con el tema: 'competencia cultural' y 'competencia cultural del traductor'. El primero fue introducido por Dell Hymes (1971) y lo define Marisa Presas (2000) como "the underlying knowledge that guides linguistic and non-linguistic action in order to make it effective" (cit. en Marcelo Wirnitzer 2007: 83). Es decir, la "competencia cultural' son los conocimientos que adquiere un individuo inconsciente, espontánea e involuntariamente durante toda su vida como consecuencia de su pertenencia a la cultura de una comunidad concreta y que lo caracterizan como miembro de esa comunidad. Por consiguiente, la competencia cultural la poseen todos los miembros de una sociedad con diferentes niveles. Por otro lado, entendemos por "competencia cultural del traductor' "el conjunto de conocimientos de naturaleza cultural que debe poseer un traductor y que abarca el conocimiento de las dos culturas que forman parte de la actividad traductora que lleva a cabo" (Marcelo Wirnitzer 2007: 83). Dicho de otra forma, es una habilidad que capacita al traductor para conocer las herramientas de la práctica traductora para poder realizar el traspaso cultural.

Aparte de estas dos competencias, el traductor de LIJ ha de contar con una tercera competencia especial que incluya un conocimiento específico de los diferentes aspectos del mundo de los niños y jóvenes. Este tipo de traductor debería poseer una sensibilidad especial que le permitiese acercarse al modo de pensar y actuar de sus supuestos lectores jóvenes.

Según lo anterior, la traducción de los marcadores culturales de LIJ requiere cierto tipo de 'intervencionismo' por parte del traductor. Este término ha sido introducido actualmente en el ámbito traductológico para referirse a la intervención, consciente o inconsciente, que realiza el traductor a la hora de enfrentarse a los problemas y dificultades de traducción como, por ejemplo, el traspaso de las referencias culturales. Marcelo Wirnitzer (2007: 145-150) expone una serie de motivos que considera claves para el intervencionismo en la traducción de LIJ:

a) El fuerte componente didáctico y pedagógico.

b) La posición periférica que ocupa la LIJ.

c) La función de la traducción, sea moralizante, moderna, etc.

d) Las características del lector y la aceptabilidad del texto meta (TM) en la CM.

e) La presencia de intermediaros entre el autor original y el texto traducido. 
f) La tradición literaria o, en otras palabras, la obligación en que se encuentra el traductor por seguir las normas vigentes y tradicionales de los cánones que se han ido creando con el tiempo y que son difíciles de romper.

En España, Isabel Pascua Febles (2000) ha sido la pionera en el uso del término 'intervencionismo' en relación con la teoría y práctica de la LIJ. Esta investigadora afirma que los traductores han empezado a ocupar posiciones más importantes en el proceso traductológico gracias a los recientes debates acerca del intervencionismo, la visibilidad y la manipulación de los traductores (cit. en Marcelo Wirnitzer 2007: 130).

Es obvio que la traducción no es una actividad neutra que se limita al mero traspaso de contenido lingüístico. Es una actividad en la que el traductor toma decisiones y deja entrever su interpretación del texto e intervención en el mismo. Por lo tanto, un texto traducido no sólo refleja el texto original (TO) y la personalidad de su autor, sino la personalidad, la experiencia lectora y el lenguaje del traductor. Como la función fundamental de la LIJ y, por consiguiente, de su traducción, es entretener al niño y adolescente, entonces las decisiones que toma el traductor y que le conducen a intervenir manipulando el texto deben tener como objetivo principal conseguir este fin, es decir, lograr que el texto sea aceptable y aceptado por los lectores infantojuveniles porque, en caso contrario, el niño no aceptará la obra y el traductor habrá fracasado en su tarea como intermediario intercultural.

\section{ANÁLISIS DE LOS MARCADORES CULTURALES}

En las II Jornadas Internacionales de Traducción e Interpretación celebradas en la Universidad de Málaga, Isabel Pascual Febles, uno de los nombres más destacados en el campo de la traducción de la LIJ, presentó una comunicación en la que señalaba la importancia que tenía la traducción de las referencias culturales dentro de la literatura infantil. Reproducimos sus palabras en relación con este tema, ya que nos servirán de punto de partida en este trabajo:

Varios ejemplos dejan claro que la no coincidencia de dos polisistemas culturales puede tener como consecuencia, en unos casos, la necesidad de explicitar en el TM ciertos marcadores implícitos del programa conceptual del autor del TO, pues de no hacerlo, quedaría incomprensible para el lector de la cultura meta y en otros casos, neutralizar e incluso omitir ciertos elementos del TO. En todo caso, la no coincidencia requiere la adaptación del TM respecto del TO, sin perder de vista el respeto a la cultura origen, la tendencia 
de internacionalismo e interculturalidad de la literatura infantil actual

(Pascua Febles 1998a: 567).

Es innegable que tanto el TO como el texto traducido (TT) no son entidades meramente lingüísticas. Cada uno de ellos supone un suceso que tiene lugar bajo ciertas condiciones sociales y temporales que conforman su esencia cultural dentro de un determinado polisistema. Incluso en los casos en que los dos polisistemas implicados -original y meta- no son muy distantes, los fenómenos culturales suponen una dificultad añadida -aparte de la lingüística, aunque ésta en un nivel inferior- debido a las diferencias existentes en cada polisistema. Cuando esa distancia es muy grande, caso de los polisistemas árabe y español, la complejidad aumenta. Y cuando el paso entre los polisistemas se efectúa en el nivel del universo literario de los niños y adolescentes, esa complejidad aumenta todavía más.

Para la clasificación y análisis de las referencias culturales, recurrimos al método propuesto por Marcelo Wirnitzer (2007), aunque adaptándolo a las necesidades de las obras analizadas ${ }^{1}$. Este método se desarrolla en cuatro fases: en la primera, se clasifican las referencias culturales en distintas categorías en función del campo lingüístico y del saber que representa cada una; en la segunda, se identifica la técnica o el procedimiento traslativo aplicado en su traducción; en la tercera, se procede a determinar la estrategia de traducción adoptada en el tratamiento de cada referencia y, en la cuarta, se determina el tipo de intervencionismo ejercido por el traductor a la hora de llevar a cabo su traducción. De acuerdo con los objetivos que perseguimos en este trabajo, aplicamos solamente las dos primeras fases mencionadas, adaptándolas a nuestro análisis: a) clasificación de las referencias culturales en categorías semánticas y b) determinación de la técnica o procedimiento traslativo adoptado en su traslado del árabe al español.

Por razones de espacio, no podremos incluir todos los elementos culturales de la obra. Por ello, nos centraremos en aquellas referencias que presentan mayores desafíos para la comprensión de la adaptación, es decir, referencias de nombres propios, fenómenos intertextuales, marcadores religiosos, creencias populares y otras referencias que aparecen en los cuentos menos de cuatro veces: concretamente dos ejemplos, uno de oficio y otro de gastronomía.

Así, pues, iniciamos el análisis comparativo-contrastivo de los marcadores culturales en la adaptación infantojuvenil, Cuentos para contar. Con él, pretendemos mostrar cómo podría haberse evitado la ambigüedad

${ }^{1}$ Este método fue aplicado en el año 2009 a un corpus mayor en mi trabajo de investigación tutelada del Máster en Traducción e Interpretación de la Universidad de Granada, que se encuentra inédito. 
en diferentes pasos de la adaptación a la hora de trasladar la referencia cultural y, por consiguiente, cómo podría haberse eliminado la oscuridad de la traducción que, consideramos, provocaría el aburrimiento de los lectores adolescentes y, como resultado final, los llevaría a abandonar la lectura.

a) Los nombres propios

Los nombres propios constituyen una de las mayores dificultades de traducción, sobre todo en los casos en que el nombre o apellido están connotados y por lo tanto influyen en la comprensión de la obra. En el cuarto cuento, Pobre... pero libre, hallamos un claro ejemplo de lo que decimos. Este cuento narra la historia de un personaje muy humilde, "عانشور الدَّنَف "Achur Ed-denf”), casado y con diez hijos, que trabaja sin descanso desde la primera hora de la mañana hasta la medianoche. La versión adaptada ha transliterado nombre ("Achur") y apodo ("Ed-denf") como si este último careciera de significado, lo que no es así. Según el diccionario árabe لسان العرب (Lessan Al-Arab), "الأنََّْ" ("Ed-denf”) significa "الكَرَضُ الملازِمُ المُخامِرُ " ("enfermedad crónica"), lo cual se corresponde perfectamente con las circunstancias miserables en que vive el protagonista. Por consiguiente, la no aclaración de la carga connotativa del apodo ha eliminado por completo el matiz que transmite en árabe.

Otro ejemplo parecido lo encontramos en el quinto cuento, La muerte de un futuwwa. El protagonista aquí, "جعلص الدنانيري" ("Gaalas Dananiri”), es un hombre que controla mediante la violencia a los egipcios humildes de su barrio, recaudando dinero de los comerciantes con el pretexto de proporcionarles protección. Lingüísticamente, el apodo "الدنانيري" ("Dananiri”) es un adjetivo árabe de relación derivado de la palabra "دنانيري" ("dinares") que es la moneda que existía antiguamente en Egipto y que sigue existiendo en varios países árabes hasta el momento. Es decir, "الدنانيري" ("Dananiri") podría traducirse al español en este contexto por "persona avariciosa, muy preocupada por el dinero". Está claro que el apodo árabe coincide muy bien con la trama de la historia protagonizada por este personaje a quien lo único que le importa es el dinero. Por eso, su transliteración al español evidencia la pérdida de su connotación implícita en árabe.

Desde nuestro punto de vista, una traducción al español de estos apodos podría servir para evitar su neutralización y transmitir sus matices tácitos en árabe. El primero, por ejemplo, podría traducirse por "Achur el miserable", mientras el segundo se traduciría por "Gaalas el extorsionador". 
b) Las referencias intertextuales

Sin duda este tipo de referencias presenta una resolución complicada, sobre todo cuando se trata de la traducción de LIJ. El primer ejemplo pertenece al primer cuento, El Gran Cheij de los derviches y el monasterio.

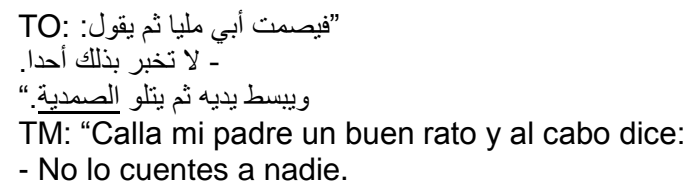

Después, con las palmas abiertas hacia lo alto, recita la Samadiyya."

"الصمدية" o "Samadiyya" es el antepenúltimo sura del Corán, libro sagrado de los musulmanes que se compone de un total de 114 capítulos o suras. No obstante, este sura no se titula así, sino "الإخلاص" o "Sura de la Adoración Pura" -según la traducción del Complejo del Rey Fahd ${ }^{2}-$. El término "Samadiyya" es una denominación tradicional 'oral' que aplicaron algunos sufíes a este sura, derivándola de una palabra que contiene la

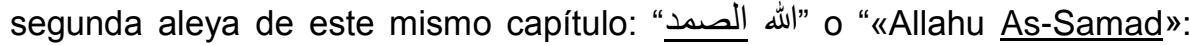
Allah, el Señor Absoluto". En la nota a pie de página, en la que se ofrece información sobre esta referencia, la traductora no precisa este hecho como acabamos de hacer, sino que pone: "Es la sura CXII del Corán que se recita para proteger de un mal" (Mahfuz 1989b: 14). Consideramos que esta falta de especificación podría confundir al lector, sobre todo si éste se animase a buscar el término "Samadiyya" en una traducción española del Corán, el cual nunca encontraría, ya que, como acabamos de indicar, no se trata del título del sura sino de una denominación tradicional oral.

Es más, la explicación que da la traductora en la nota incluye información ambigua: "Es la sura CXII del Corán que se recita para proteger de un mal" (ibíd.). Por un lado, utiliza los números romanos para identificar el sura, lo que podría resultar difícil de descifrar para un lector adolescente. Por otro lado, llama la atención el hecho de que la información que recoge la nota no se ajuste del todo a la realidad, ya que este sura no se recita especialmente para protegerse contra el mal, como afirma la traductora. En nuestra opinión, podría haberse evitado esta ambigüedad si se hubiese optado por una de las siguientes alternativas: o quitar la palabra "Samadiyya" y poner la traducción al español de su título original, tal y como

\footnotetext{
${ }^{2}$ Para la traducción y aclaración de significados de referencias relacionadas con El Corán hemos utilizado la traducción oficial realizada por El Complejo del Rey Fahd Para la Edición del Noble Corán, proyecto iniciado por Arabia Saudí, que está disponible en línea en el enlace siguiente: http://www.qurancomplex.org/Display.asp?section=7\&l=esp\&f=nobza01
} 
aparece en cualquiera de las traducciones españolas del Corán, o mantener esa palabra, como ha hecho la traductora, pero explicitando más información en la nota a pie de página o, incluso, dentro del mismo texto lo que facilitaría su comprensión a los lectores adolescentes. Nosotros optamos por la segunda opción, igual que la traductora, pero aclarando más para evitar confusiones y ambigüedades.

El segundo ejemplo se halla en el segundo cuento, Um Zaki.

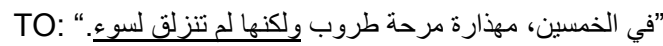

TM: "Um Zaki es una cincuentona alegre y jovial. Le gusta bromear, pero nunca hizo mal a nadie."

En este caso, la traducción neutraliza demasiado el sentido de la referencia al provocar una variación semántica entre el TO y el TM. La traducción literal de "و لكنها لم تنزلق لسوء" es "pero no se ha resbalado en nada malo"; sin embargo, el significado cultural implícito de esta expresión implica más cosas. "تنزلق لسوء" o "resbalarse en lo malo" es una expresión que se emplea siempre en árabe para referirse a las relaciones sexuales que puede tener alguien sin estar casado, lo cual representa un gran tabú en la tradición árabe. En el texto español la traductora ha transmitido un significado más neutro, que pierde el sentido moral de la expresión; es más, en la traducción se entiende que "Um Zaki" no hace daño 'físico' a nadie, lo que no es del todo exacto pues el texto original se refiere al 'mal' que podría cometer una persona hacia sí misma y no al 'daño' que cometería hacia otra persona.

Proponemos la siguiente traducción para esta referencia: "Um Zaki es una cincuentona alegre y jovial. Le gusta bromear, pero nunca ha sido una mujer facilona"; pensamos que de este modo se mantiene el contenido esencial de la referencia cultural, pero, al mismo tiempo, adaptándolo a los lectores adolescentes occidentales para que puedan entenderlo, dada la diferencia cultural entre ambas sociedades.

c) Las referencias religiosas

Se consideran parte inseparable del patrimonio lingüístico árabe, ya que los árabes (y en este caso los egipcios) siempre introducimos en el discurso oral palabras y frases relacionadas con la religión, hecho que es muy habitual en los diálogos de las historias de Cuentos para contar. El primer ejemplo aparece en el segundo cuento, Um Zaki: 
TO: وجلجلت زغرودة وارتفع صوت مبتهلا:

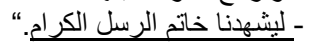

TM: "Retumba un grito de júbilo y una voz implora:

- Que el sello de los santos profetas nos sirva de testigo..."

En este caso, la unidad de traducción ha sufrido en la versión española una pérdida parcial importante. La traductora ha confundido la categoría morfológica del vocablo árabe "خاتم", adjetivo que significa "último", considerándolo como un sustantivo que significa "sello". Debido a esta misma desviación, se ha provocado en el TM una generalización de la connotación cultural de dicha unidad al emplear la transposición de 'el todo por la parte', convirtiendo al Profeta Mohammad (Mahoma) en "Ios santos profetas".

La importancia de esta referencia radica en los preceptos dogmáticos de los musulmanes que sitúan a su Mensajero en el mejor compañero y testigo de cualquier acto o acontecimiento social.

Así pues, nuestra propuesta para la transferencia de esta unidad sería: "Con la venia y la bendición del último de los nobles profetas". También, podría añadirse el nombre mismo del Profeta Mohammad para aclarar más esta condición, explicitando así una información implícita en el texto original. un futuwwa.

El segundo ejemplo se encuentra en el quinto cuento, La muerte de

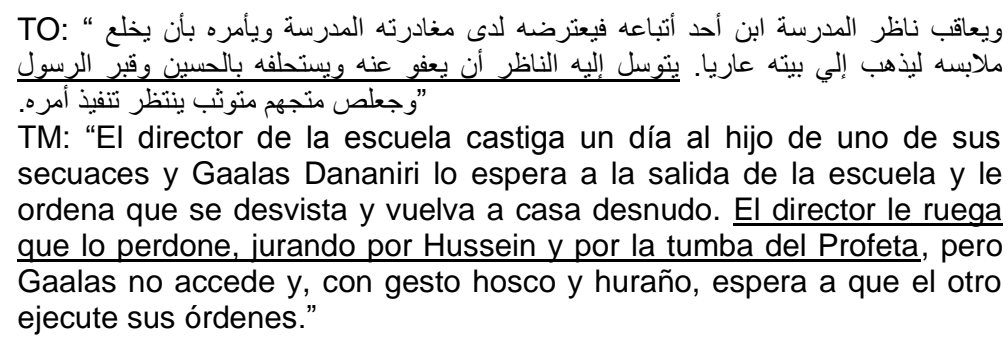

Esta vez se detecta un calco literal del árabe al transliterar el nombre "الحسين" en español "Hussein". La traducción literal transmite lo que dice el original, pero la transliteración "Hussein", que va sin aclaración, podría provocar cierta ambigüedad. En este contexto, "الحسين" o "Hussein" se refiere al nieto del Profeta de los musulmanes, Mohammad, a cuya mención recurren algunos egipcios humildes en sus juramentos; es decir, en vez de jurar por Dios, lo hacen por la tumba de "الحسين" ("Hussein"), la cual se encuentra en El Cairo. No obstante, en la traducción se cita a "Hussein" sin dar ninguna pista de quién es o de cómo es la tradición de la que forma 
parte. Además, el nombre va precedido en árabe de un artículo determinado -"Al-Hussein"-, que no aparece en la transliteración española. En nuestra opinión, podría haberse evitado la ambigüedad explicitando alguna información dentro del propio texto como, por ejemplo, "El director le ruega que lo perdone, jurando por la tumba del Profeta Mohammad y por la de su nieto, Al-Hussein".

d) Referencias relacionadas con las creencias populares

Tienen que ver con algunas convicciones muy arraigadas en la cultura egipcia. Encontramos un claro ejemplo en el tercer cuento, La casa de los perros y los gatos.

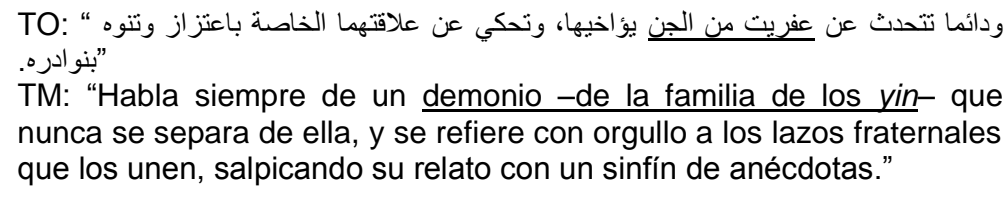

La traductora introduce el árabe "لجن" o "yin (demonio)" en el texto de la adaptación sin ninguna aclaración, resaltándolo apenas con la cursiva, lo que podría provocar cierta ambigüedad en el lector adolescente, o incluso en cualquier lector, ya que se trata de una palabra extraña. Además, el lector corre el riesgo de confundir esta palabra con otra homógrafa procedente de la filosofía china ('yin' y su opuesto 'yang'). El único significado que recoge el Diccionario de la RAE de la palabra "yin" es el relacionado con la filosofía oriental, hecho que corrobora Internet donde se recoge también este mismo sentido. A nuestro juicio, una traducción que reflejaría el sentido de la referencia árabe sería: "Habla siempre de un duende de la familia de los genios...", ya que la palabra "الجن" o "yin" no comporta ninguna carga cultural, sino que lo que prima en este ejemplo es la idea misma. En otras palabras, lo que importa trasladar al español aquí es el 'significado' y no el 'significante'.

e) Otros elementos culturales (oficio y gastronomía)

Como hemos mencionado más arriba, incluimos en esta categoría las referencias de áreas temáticas que aparecen menos de cuatro veces a lo largo de los cuentos. Presentamos dos ejemplos: uno de oficio y otro de gastronomía. El primero pertenece al segundo cuento, Um Zaki.

TO: "ربنا يحفظك و أشوفلك ماشطة،

TM: "- Que Dios te proteja y un día te veré de masajista en un instituto de belleza." 
El término "ماشططة" ("peluquera”, término casi en desuso) se refiere al oficio, ya antiguo, de las peluqueras privadas que iban a las casas de las clientas para peinarlas así como también, en ocasiones, al de las depiladoras (esta última acepción es más cultural que lingüística). La acepción que recogen los diccionarios es la relacionada con 'peinar el pelo'. En el cuento, la protagonista, 'Um Zaki', usa esta palabra cuando se dirige al chico mientras éste le da un buen masaje de espalda; entonces, para recompensarle espiritualmente, incluye una fórmula muy frecuente en el discurso oral egipcio, a modo de plegaria a Dios, para que llegue a ser un buen "ماشطة" o "peluquera". Pero, influida por el contexto, la traductora confunde esta palabra con "masajista": "Que Dios te proteja y un día te veré de masajista", por lo que se pierde tanto el significado lingüístico como la connotación cultural. Por otra parte, esta misma desviación dio lugar a la sobretraducción en el texto español añadiendo "en un instituto de belleza". De este modo, lo primero que se nos viene a la mente es la imagen de un 'centro de belleza', un establecimiento muy moderno que nada tiene que ver con el momento ni con el lugar donde transcurre el cuento. En la contracubierta de Cuentos para contar, Juan Goytisolo informa a los lectores de que las historias incluidas representan la infancia del narrador. Si tenemos en cuenta que Mahfuz nació en el año 1911 en un barrio popular de El Cairo, podremos comprobar el gran desfase cultural que se ha cometido al añadir esa información. Por consiguiente, en la traducción no solamente se pierde el significado y la connotación cultural de la referencia, sino que se lleva al lector fuera del tiempo y del espacio donde transcurren los hechos del cuento. El segundo y último ejemplo de marcadores culturales se halla en el cuarto cuento, Pobre...pero libre.

TO: “يحتقن بالحسرات إذا رأى الناعمين في المقهي أو تطايرت إلى أنفه رائحة التقلية."

TM: "El hombre se indigna y lamenta su suerte cuando ve a gentes acomodadas sentadas en el café o llegan a su nariz olores a frituras."

Esta referencia es muy típica en la gastronomía egipcia, ya que se relaciona con un plato de verdura muy popular: "الملوخية" ("malva de los judíos"). Para elaborar dicho plato se precisa una especie de sofrito llamado "التقلية" ("ajo machacado y frito con comino en aceite"). Como se observa, es algo completamente diferente de lo que dice la traducción española, pues el término "frituras" se refiere a cualquier alimento frito. Por otra parte, del citado sofrito, y debido a otros condimentos, se desprende un olor peculiar que tradicionalmente se asocia con abrir el apetito a los comensales. Por consiguiente, se produce en la versión española una pérdida parcial de la connotación tanto gastronómica como social que lleva implícita la referencia árabe. 
Una alternativa en este caso podría haber sido explicitar alguna información dentro del texto, mencionando los ingredientes de esta fritura y su relación con el plato principal, "الملوخية" ("malva de los judíos"), porque la gastronomía puede ser también una fuente de conocimiento para los lectores adolescentes. Como segunda opción, podría neutralizarse dicha referencia empleando el procedimiento técnico de transposición de 'parte por el todo', haciendo hincapié en la frustración que provocaba oler la comida en nuestro personaje hambriento. La traducción sería: "El hombre se indigna y lamenta su suerte cuando ve a gentes acomodadas sentadas en el café o cuando le llegaban los aromas de la comida".

\section{CONCLUSIÓN}

En este estudio hemos examinado la importancia de los marcadores culturales en la traducción literaria, sobre todo en el caso de la traducción de la LIJ. Asimismo, hemos comprobado cómo este aspecto no se ha tenido suficientemente en cuenta en la adaptación Cuentos para contar debido al uso de la transliteración, sin ir acompañada de suficientes explicaciones, y a las variaciones semánticas que llevan unas veces a generalizar el significado y otras veces a neutralizarlo demasiado. Por ello, nos encontramos ante una traducción en la que predomina la ambigüedad en el traspaso de los elementos culturales.

Oittinen (2000: 74; 2006: 43) sostiene que, al encontrarse ante una 'solución exótica', el niño puede aburrirse y dejar de leer el texto traducido, ya que estamos hablando de un tipo de lector con características muy determinadas y en proceso de formación, por lo que advierte de las consecuencias que esto puede tener para los futuros hábitos de lectura del niño. En el análisis realizado, hemos podido corroborar la advertencia de la estudiosa finlandesa.

Es cierto que en los últimos años se ha extendido en el campo de la LIJ el fenómeno del internacionalismo y la multiculturalidad, cuya finalidad consiste en informar a los niños y adolescentes sobre otras vidas, culturas, costumbres, pueblos, etc., con objeto de fomentar la universalidad de la condición humana. De esta manera, el niño se da cuenta de que las personas que aparecen en los libros extranjeros, a pesar de vivir en pueblos y países muy lejanos e inmersos en culturas muy distintas de la suya, comparten con él los mismos problemas y sentimientos, lo cual reforzará su sensación de pertenencia al conjunto de la humanidad: todos pertenecemos a un solo 'pueblo', todos perseguimos los mismos fines. Y así se crea lo que Pascua Febles (1998b: 17) denomina "«multiculturalismo»: crear un niñolector multicultural a través de libros de diferentes países, conociéndose gracias a las traducciones". 
La iniciativa llevada a cabo por la Editorial Libertarias/Prodhufi de introducir a un escritor egipcio, y, más aún, a un autor del calibre de Mahfuz, en el mundo infantojuvenil español nos parece una respuesta al llamamiento que hace Carmen Valero Garcés de publicar traducciones de obras de otras culturas que no pertenezcan al mundo anglosajón, ya que, según esta autora, en España "la temática de los libros traducidos, prácticamente el $90 \%$ refleja la sociedad occidental y un alto porcentaje se inserta dentro de la cultura anglosajona" (2000: 248-249, cit. en Morales López 2008: 16). Es más, nos parece un paso sin precedentes para alcanzar el objetivo de presentar el mundo árabe, concretamente el egipcio, a los niños y adolescentes españoles, con todas sus diferencias (costumbres, nombres, comidas, etc.), para que poco a poco vayan conociéndolo y les ayude a fomentar el entendimiento intercultural y universal.

Ahora bien, no estamos seguros de hasta qué punto podría considerarse esta iniciativa editorial un éxito. Es verdad que la corriente internacionalista va muy ligada en traducción a lo que se ha venido llamando extranjerización o exotización (Venuti 1995, 2008); sin embargo, desde nuestro punto de vista, en el caso que nos ocupa la exotización se ha practicado de forma extrema.

Como hemos señalado al principio de este trabajo, el original árabe, (Hikayat haratina), tuvo una primera traducción para adultos Historias de nuestro barrio-, a la que siguió poco después la adaptación infantojuvenil, Cuentos para contar. Las dos traducciones -primera para adultos y segunda para adolescentes- se diferencian solamente en los aspectos paratextuales: en la adaptación se añadieron ilustraciones en los cinco cuentos escogidos; la portada y la contracubierta sufrieron un cambio para adecuarse al público meta; el tamaño y fuente de la letra cambió además del título de la obra, etcétera. Sin embargo, no se produjo ninguna modificación en el material lingüístico. Dicho de otro modo, ni el nivel ni el registro lingüístico de la versión española para adultos -que es resultado directo del traslado al español de un nivel y registro lingüístico semejantes del original árabe que va dirigido a lectores adultos árabes- han sufrido cambio alguno en la versión para adolescentes. De todo lo cual parece desprenderse que lo que vale para un público lector adulto también vale para un público lector adolescente con características distintas, lo que en nuestra opinión no es así.

No cabe duda de que el hecho de introducir al Nobel egipcio, Naguib Mahfuz, en el mundo infantojuvenil español, y recurrimos aquí a la expresión usada por Arias Torres para describirlo, "es un éxito sin parangón" porque lo que se lee "durante la infancia y la juventud marca el resto de la vida" (2003: 129). Hay que señalar además que esta oportunidad que se le ha ofrecido al público adolescente español no tiene antecedentes, 
que sepamos, por lo menos en lo concerniente a escritores egipcios que no pertenezcan al mundo de la LIJ. Ahora bien, para que esta oportunidad cumpla su verdadero objetivo, que es dar a conocer a este Premio Nobel a un público extranjero menor de edad, las peculiaridades de este público deberán primar sobre cualquier otro aspecto a la hora de efectuar la traducción para que no se produzca un efecto contrario al deseado.

En la comunicación mencionada al principio, Isabel Pascua Febles confirma que:

\begin{abstract}
Está claro que no existen "recetas mágicas", ni siquiera normas generales de cómo traducir estos marcadores en los cuentos infantiles. Una de nuestras conclusiones es que su análisis fuera de la situación comunicativa y el programa conceptual del autor del texto no aporta resultados concretos, pues todo depende del valor comunicativo de cada referencia concreta, de ahí la importancia del contexto cultural, debiéndose enfocar todo hacia el programa conceptual del autor y la aceptabilidad del TM en la cultura meta, los dos principios fundamentales que deben regir cualquier traducción comunicativa (Pascua Febles 1998a: 567).
\end{abstract}

Es innegable que el libro es un medio fundamental para acceder al conocimiento y para un adolescente es mucho más enriquecedor porque le abre las puertas a otros mundos, ampliando su ámbito cultural, promoviendo y desarrollando en él más comprensión y sensibilidad hacia lo ajeno. Sin embargo, cuando se traduce para él, lo que debe tenerse en cuenta es la aceptabilidad del TT en la nueva cultura según los conocimientos del adolescente. Puede mantenerse lo exótico para darle a los jóvenes la oportunidad de enfrentarse a lo extranjero y diferente, según promueve el fenómeno del internacionalismo y la multiculturalidad en la LIJ, pero hasta cierto punto, permitiéndose a la vez las adaptaciones o modificaciones que el traductor considere oportunas o necesarias, y ambas opciones tienen que tener como objetivo último que el texto consiga su meta; que resulte aceptable y aceptado por su lector.

\title{
REFERENCIAS BIBLIOGRÁFICAS
}

ARIAS TORRES, Juan Pablo, "Mercado y traducción: Naguib Mahfuz para niños". En: Isabel Pascua Febles et al. (coords.), Traducción y literatura infantil. Las Palmas de Gran Canaria: Universidad Las Palmas de Gran Canaria, 2003, pp.117-132.

BÖDEKER, Birgit y Katrin FREESE, "Die Übersetzung von Realienbezeichnungen bei literarischen Texten: Eine Prototypologie". En: Textcontext, 2(3), 1987, pp. 137-165. 
Franco AIXELÁ, Javier, "Culture-specific Items in Translation". En: Román Álvarez y M. Carmen-África Vidal (eds.), Translation, Power, Subversion. Clevedon: Multilingual Matters, 1996, pp. 52-78.

GONZÁLEZ CASCALLANA, Belén, "Translating Cultural Intertextuality in Children's Literature". En: Jan Van Coillie y Walter P. Verschueren (eds.), Children's Literature in Translation: Challenges and Strategies. Manchester: St. Jerome, 2006, pp. 97-110.

Hermans, Theo, "On Modeling Translation: Models, Norms and the Field of Translation". En: Livius, 4, 1993, pp. 69-88.

HeRRERo, Leticia, "Sobre la traducibilidad de los marcadores culturales". En: Andrew Chesterman et al. (eds.), Translation in Context: Selected Contributions from the EST Congress, Granada, 1998. Amsterdam, Pliladelphia: John Benjamins, 2000, pp. 307-316.

IBN MANZUR, [en línea] Diccionario árabe Lisan Al-Arab (معجم لسان العرب). Disponible en línea en <http://www.baheth.info/index.jsp>

KOLLER, Werner, Einführung in die Übersetzungswissenschaft. Heidelberg: Wiesbaden, 1992.

KRISTEVA, Julia, Séméiotiké: Recherches pour une sémanalyse. Paris: Éditions du Seuil, 1969.

, "Bajtín, la palabra, el diálogo y la novela". En: Disiderio Navarro (ed.), Intertextualité: Francia en el origen de un término y el desarrollo de un concepto. La Habana: Editorial Casa de Las Américas, 1997, pp. 1-24.

MARCELO WIRNITZER, Gisela, Traducción de las referencias culturales en la literatura infantil y juvenil. Frankfurt: Peter Lang, 2007.

MAYORAL, Roberto, "La explicitación de información en la traducción intercultural". En: Amparo Hurtado Albir (ed.), Estudis sobre la traducció. Castelló: Universitat Jaume I, 1994, pp. 73-96.

MAYORAL, Roberto y Muñoz, Ricardo, "Estrategias comunicativas en la traducción intercultural". En: Purificación Fernández y José María Bravo (coords.), Aproximaciones a los estudios de traducción. Valladolid: Servicio de Apoyo a la Enseñanza, Universidad de Valladolid, 1997, pp. 143-192.

MORALES LóPEZ, Juan Rafael, La exotización en la traducción de la Literatura Infantil y Juvenil: el caso particular de las traducciones al español de las novelas infantojuveniles de José Mauro de Vasconcelas. Las Palmas de Gran Canaria: Universidad de Las Palmas de Gran Canaria, 2008.

NeWmaRK, Peter, Approaches to Translation. Oxford: Pergamon Press, 1982. 
NIDA, Eugene A., Toward a Science of Translating: With Special Reference to Principles and Procedures involved in Bible Translating. Leiden: Brill, 1964.

NORD, Christiane, "It's Tea-Time in Wonderland: culture-markers in Fictional Texts". En: Heiner Pürschel et al. (eds.), Intercultural Communication. Proceedings of the $17^{\text {th }}$ International L.A.U.D. Symposium Duisburg 1992. Frankfurt etc.: Peter Lang, 1994, pp. 523-538.

OITTINEN, Riitta, Translating for Children. New York: Garland, 2000. , "No Innocent Act: On the Ethics of Translating for Children". En: Jan Van Coillie y Walter P. Verschueren (eds.), Children's Literature in Translation: Challenges and Strategies. Manchester: St. Jerome, 2006, pp. 35-46.

PASCUA FEBLES, Isabel, "Contexto cultural en la traducción de cuentos infantiles". En: Leandro Félix Fernández y Emilio Ortega Arjonilla (coords.), II Estudios sobre traducción e interpretación: Actas de las II Jornadas Internacionales de Traducción e Interpretación de la Universidad de Málaga. Málaga: Universidad de Málaga, 1998a, pp. 567-571.

, La adaptación en la traducción de la literatura infantil. Las Palmas de Gran Canaria: Universidad de Las Palmas de Gran Canaria, 1998b.

PAZ, Octavio, Traducción: literatura y literalidad. Barcelona: Tusquets, 1971.

PRESAS, Marisa, "The Translator between two Cultures: Cultural Competence as a Component of Translation Competence". En: $28^{\text {th }}$ Annual Research Symposium "Translations: Paradigms of Linguistic and Cultural Transformation". Coleraine: University of Ulster, 2001.

SAMY GAMAL-ELDIN, Sherine, Las referencias culturales e intertextuales como recursos de exotización en traducciones adaptadas al público infantojuvenil: el caso de Cuentos para contar, de Naguib Mahfuz (Proyecto de Investigación Fin de Máster, inédito). Universidad de Granada, 2009.

VALERO GARCÉS, Carmen, "Internacionalización y multiculturalismo de la LIJ en castellano: La importancia de la traducción". En: Veljka Ruzicka Kenfel et al. (eds.), Literatura infantil y juvenil: tendencias actuales en investigación. Vigo: Universidad de Vigo, 2000, pp. 241-254.

VENUTI, Lawrence, The Translator's Invisibility: A History of Translation. London: Routledge, 1995/2008.

WITTE, Heidrun, "Translation as a means for a better understanding between cultures". En: Cay Dollerup y Annette Lindegaard (eds.), Teaching Translation and Interpreting 2. Insights, Aims, Visions. Amsterdam: John Benjamins, 1994, pp. 69-75. 
OBRAS UTILIZADAS EN EL ANÁLISIS

MAHFUZ, Naguib, Cuentos para contar. Trad. María Rosa de Madariaga. Madrid: Libertarias/Prodhufi, 1989 b.

Hikayat haratina. El Cairo: Maktabat Misr, 1983 [1a ed.: El Cairo: Maktabat Misr, 1975].

, Historias de nuestro barrio. Trad. María Rosa de Madariaga. Madrid: Libertarias/Prodhufi, 1989a. 